\title{
An improved adaptive wavelet shrinkage for ultrasound despeckling
}

\author{
P NIRMALA DEVI ${ }^{1, *}$ and R ASOKAN ${ }^{2}$ \\ ${ }^{1}$ Department of Electronics and Communication Engineering, Kongu Engineering \\ College, Perundurai 638 052, India \\ ${ }^{2}$ Department of Electronics and Communication Engineering, Kongunadu College of \\ Engineering and Technology, Thottiyam 621 215, India \\ e-mail: nirmaladevi@kongu.ac.in; asokece@yahoo.com
}

MS received 16 May 2013; revised 24 September 2013; accepted 24 February 2014

\begin{abstract}
Ultrasound imaging is the most widely used medical diagnostic technique for clinical decision making, due to its ability to make real time imaging for moving structures, low cost and safety. However, its usefulness is degraded by the presence of signal dependent speckle noise. Several wavelet-based denoising schemes have been reported in the literature for the removal of speckle noise. This study proposes a new and improved adaptive wavelet shrinkage in the translational invariant domain. It exploits the knowledge of the correlation of the wavelet coefficients within and across the resolution scales. A preliminary coefficient classification representing useful image information and noise is performed with a novel inter-scale dependency measure. The spatial context adaptation of the wavelet coefficients within a subband is achieved by a local spatial adaptivity indicator, determined by using a truncation threshold. A weighted signal variance is estimated based on this measure and used in the determination of a subband adaptive threshold. The proposed thresholding function aims to reduce the fixed bias of the soft thresholding approach. Experiments conducted with the proposed filter are compared with the existing filtering algorithms in terms of Peak-Signal to Noise Ratio (PSNR), Mean Square Error (MSE), Structural Similarity Index Measure (SSIM), Equivalent Number of Looks (ENL) and Edge Preservation Index (EPI). A comparison of the results shows that the proposed filter achieves an improvement in terms of quantitative measures and in terms of visual quality of the images.
\end{abstract}

Keywords. Wavelet; translation invariance; inter and intra scale dependency; speckle; adaptive thresholding; ultrasound images.

${ }^{*}$ For correspondence 


\section{Introduction}

Medical image scanning is a vital step for the treatment and diagnosis of various diseases. Ultrasonography is a widely used diagnostic tool in modern medicine for visualization of soft tissues such as muscles and internal organs of the human body. Ultrasound imaging technique is used in medical diagnosis because of the following advantages: non-invasive, low cost and the capability of forming real time imaging (Arsenault \& April 1976; Suetens 2002). However, the presence of signal dependent noise called speckle degrades the quality of ultrasound imaging and so it becomes unsuitable for further processing. Nevertheless, image denoising is an essential pre-processing step in image processing applications.

Speckle, a multiplicative noise pattern, gets added to the digital images during its capturing and transmission stages. Speckle noise results due to the constructive-destructive interference of the coherent ultrasound pulses that are backscattered from the tiny biological tissues. Ultrasound images are formed by transmitting a sound wave and by receiving the echoes that are reflected from the biological tissues. They give rise to a granular pattern in the imaging data, which may obscure some of the important image features (Goodman 1976; Xie et al 2002). So it becomes a complicated process for the physician to make the diagnosis.

Two approaches for speckle reduction were found in the literature: (i) Spatial domain and (ii) Transform domain approaches. The spatial domain approaches include those of Lee, Frost and Kuan Filters (Kaur et al 2011). In Lee and Kuan filters, the output of the processed pixel is the average intensity of the pixels within a local window. Frost filter produces an output by forming an exponential kernel. Recent investigations in the literature (Achim et al 2001; Chen \& Suter 2004; Chen et al 2009; Kalaivani Narayanan \& Wahidabanu 2009; Viswa \& Sharma 2012; Gao 1998) illustrate a significant use of wavelet transform for denoising, called nonlinear filtering. Wavelet denoising attempts to remove noise and preserve the signal details irrespective of their frequency content.

Denoising by thresholding in wavelet domain was developed by (Donoho 1995). However, the Discrete Wavelet Transform (DWT) lacks translation invariance. The Stationary Wavelet Transform (SWT) overcomes this drawback by removing the down samplers and upsamplers in the wavelet transform. SWT is a redundant scheme as the output of each level has the same number of samples as the input (Coifman \& Donoho 1995; Matsuyama et al 2012; Gyaourova et al 2002). Kim \& Ra (2005) proposed an image enhancement technique based on a multiresolution approach in the translational invariant domain. Their algorithm stipulated directional filtering and noise reducing procedure for the multiresolution data. Directional filtering was performed by analysing the structural feature of each pixel through the eigen values. The reduction of wavelet coefficients belonging to homogenous regions decrease speckle noise. Zhang et al (2005), prescribed an image restoration approach exploiting inter and intra scale dependency along the wavelet coefficients using linear minimum mean square estimation. Pizurica et al $(2002,2003)$ in their work presented a denoising algorithm considering the magnitude, evolution of the co-efficients at various scales and the clustering of the large magnitude coefficients near the edge pixels. Various adaptive thresholding schemes have been introduced in recent studies as in (Xu et al 2009; Sudha et al 2009; Bhuiyan et al 2009; Yang et al 2011; Ismail \& Khan 2012; Pižurica et al 2001). Qin et al (2010) posited an improved thresholding method to reduce the fixed-bias of soft thresholding.

Edges are high frequency components that distinguish the constant gray levels and the boundaries. By setting a high threshold to remove the high energy noisy coefficients, edge pixels are also removed. Without edge information, the image appears to be blurred so that it becomes 
difficult for processing such as segmentation and classification (Yoo et al 1999). Hence the performance of denoising algorithm is also dependent on its ability to preserve the edge features in an image after reconstruction. This study proposes a Shift Invariant Improved Adaptive Thresholding Function (SIIATF). It helps to remove the noisy coefficients thereby simultaneously preserving the fine details of the image. The proposed algorithm aims to improve the performance of Qin et al (2010) as per their future directions, by incorporating the secondary wavelet properties and also modified further to reduce the number of zero coefficients around the threshold. A new intra scale dependency measure is proposed for the preliminary grouping of significant image and noisy pixels. The weighted signal variance is estimated by exploiting the intra scale dependency of the wavelet coefficients. The weight measure is determined using a new truncation threshold. The shrinking of wavelet coefficients is achieved through a subband adaptive threshold.

This paper includes the following sections. Section 2 explains intra-scale and inter-scale dependencies among the wavelet coefficients. Section 3 describes the proposed new and improved adaptive thresholding function. Section 4 deliberates the proposed noise removal algorithm and section 5 discusses the performance improvement of the proposed method over the existing techniques.

\subsection{Formation of speckle}

The block diagram of ultrasound imaging system is shown in figure 1 .

The ultrasound images are constructed by capturing the returned echo signals from tissues at the surface of the transducers. Conversion of ultrasonic RF mechanical wave into electrical signal is carried out by the transducer. Echoes that are returned from tissue are collected by ultrasound probes. There is a group of transducers that are simultaneously activated at each spatial direction, to generate a raw line signal (stick) for raster image construction. These sticks are demodulated and compressed logarithmically for reducing their dynamic range, to suit the commercial display. The Cartesian image is constructed by a scan conversion process from the sampled sticks (Kalaivani Narayanan \& Wahidabanu 2009).

Speckle, a multiplicative noise pattern, gets added to the digital images during its capturing and transmission stages. Speckle noise results due to the constructive-destructive interference of the coherent ultrasound pulses that are backscattered from the tiny biological tissues. They

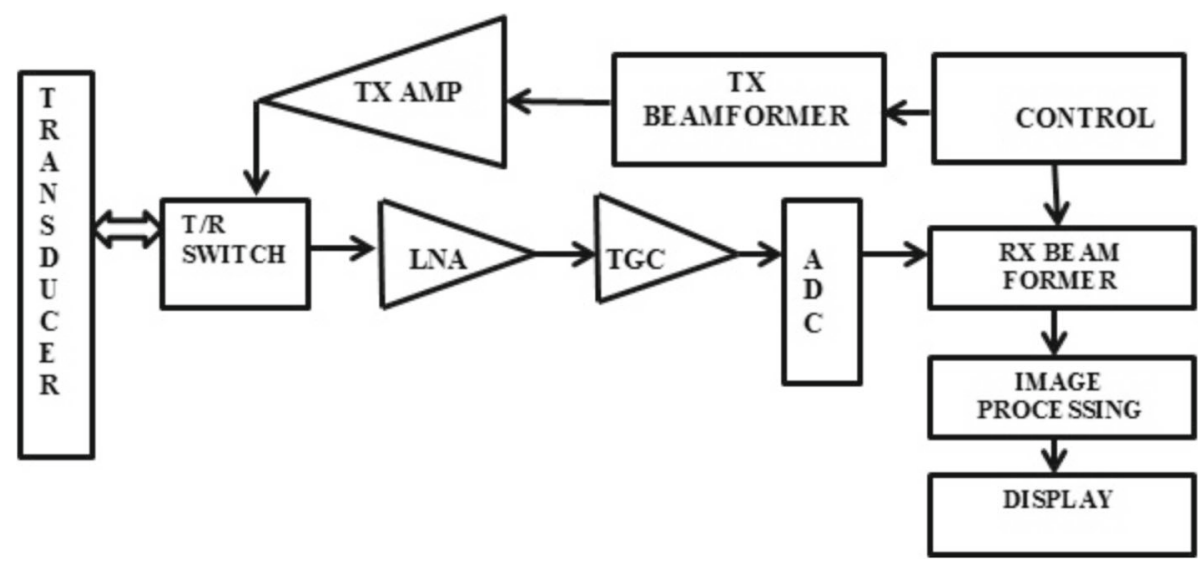

Figure 1. Block diagram of ultrasound imaging system. 
give rise to a granular pattern in the imaging data. This may obscure some of the important image features and hence it becomes a tedious process for the physician to make the diagnosis. The nature of the speckle pattern can be categorized according to the number of scatterers per resolution cell, spatial distribution and the characteristics of the imaging system itself (Kalaivani Narayanan \& Wahidabanu 2009).

The generalized model of speckle assumes a multiplicative pattern as given below:

$$
g(x, y)=f(x, y) \bullet v(x, y),
$$

where $g(x, y)$ is the speckled image, $f(x, y)$ is the original image and $v(x, y)$ is the speckle noise.

In this study, to verify the quantitative performance of the algorithm, an artificial speckle is introduced in the images using the MATLAB command as given in equation

$$
\mathrm{J}=\text { imnoise }(\mathrm{I}, \text { 'speckle', v), }
$$
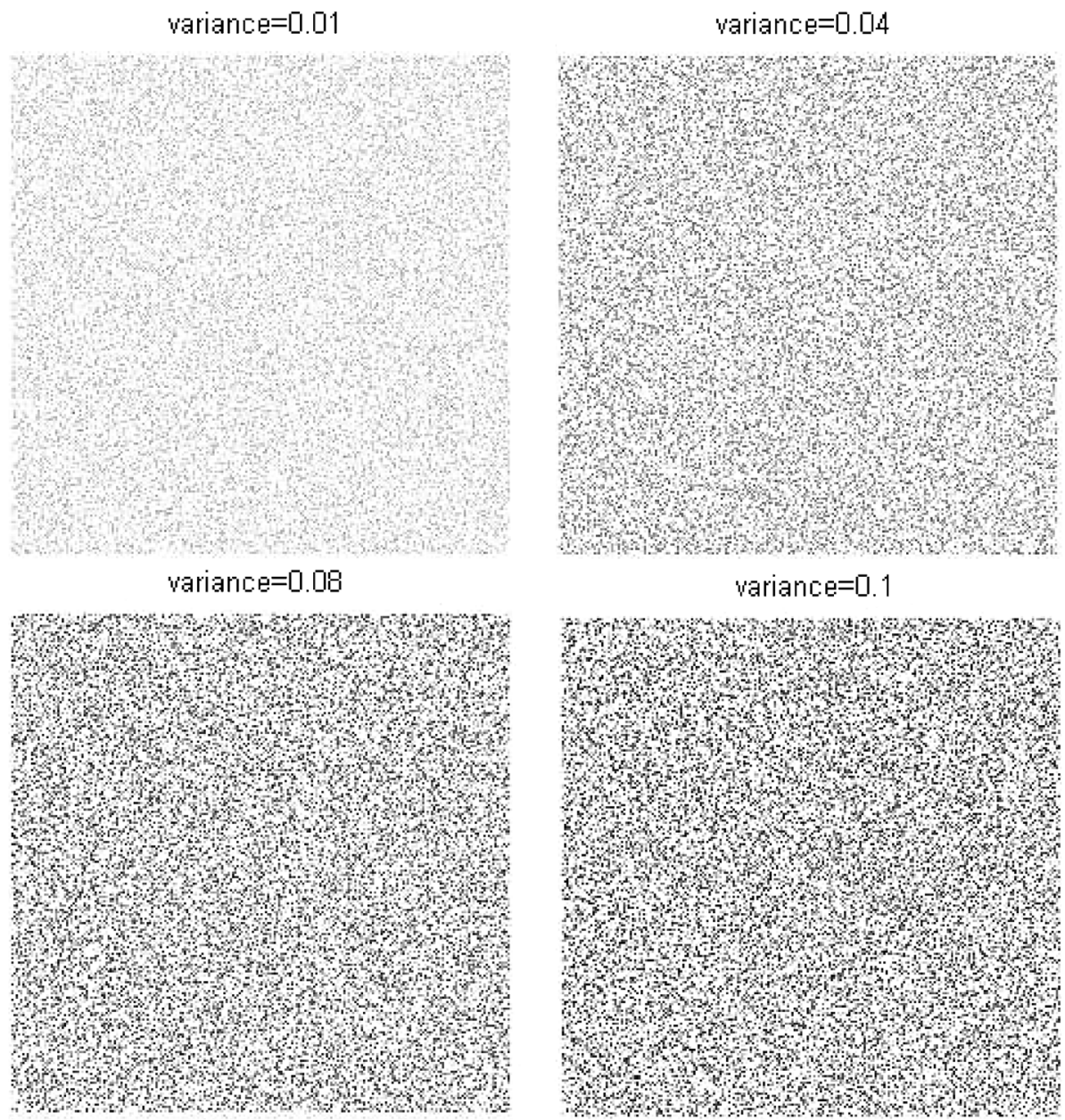

Figure 2. Artificial speckle introduced for different noise variances. 
where I is the original image and $\mathrm{v}$ is the noise variance. This command adds multiplicative noise to the image I. The generated speckle by simulation using Eq. (2) is given in figure 2.

\section{Proposed wavelet shrinkage algorithm}

\subsection{Wavelet speckle denoising}

Wavelet transform is extensively used by the researchers for noise removal because it spreads noise among all the coefficients and compresses the essential image details into fewer numbers of coefficients. Hence, fine details and edges can easily be identified, even if there is a large amount of noise present in the images. The procedure for wavelet denoising algorithm can be summarized by, computing the $2 \mathrm{D}$ wavelet transform, thresholding the coefficients and reconstructing the image using inverse wavelet transform.

In the wavelet domain, the larger magnitude coefficient is said to be an important image data and a smaller magnitude coefficient to be an insignificant image data. Also, small image details are revealed at finer scales. Preservation of fine details in an image is accomplished by exploiting the dependencies between two adjacent scales called inter scale dependency and also by the correlation among the coefficients in the same scale called intra scale dependency. To study the inter scale dependency, the subbands at various scales should have the same size. This property of shift invariance is lacking in discrete wavelet transform. For this reason, a Stationary Wavelet Transform (SWT) which inherits the property of translation invariance is used in this paper.

\subsection{Shift invariant improved adaptive wavelet thresholding function (SIIATF)}

Here, a new and improved adaptive wavelet shrinkage algorithm with subband adaptive threshold for the removal of speckle noise in ultrasound images is proposed.

A non-decimated wavelet transform is used to decompose the noisy image into the required number of levels. Each level consists of one approximation $(L L)$ and three detail $(L H, H L, H H)$ images, with an equal number of coefficients in all the levels. The notation for the detail coefficient is $W_{j}^{D}=\left\{W_{1}^{D}, W_{2}^{D} \ldots \ldots \ldots \ldots W_{J}^{D}\right\}$ where $J$ is the total number of decomposition levels.

2.2a Determination of mask using inter scale dependency: The first step in the proposed algorithm is to determine a mask for each detail subband to identify the significant pixels of the image. The mask contains a value of either 0 or 1 , depending on the degree of significance of each pixel. The degree of significance is determined using a rule.

The mask for each subband image with $\mathrm{M}$ number of coefficients is denoted as $\hat{I}_{i, j}$. The mask is estimated using a simple classification technique based on the following empirical observations: at positions of significant image details the wavelet coefficients increase in magnitude across the scales and at positions of noise, their value tends to decrease as the resolution scale increases (Liu \& Moulin 2001). This inter-scale and intra-scale dependency of the wavelet coefficients has been exploited by many authors for noise removal in digital images in different ways (Puvanathasan \& Bizheva 2007). Xu et al (2009) multiplied the adjacent wavelet scales to sharpen the edge structures while weakening noise. Sadler \& Swami (1999) analysed the product of multiscale wavelet coefficients and used them to step detection and estimation. Pizurica et al (2003) used a method for signal of interest classification using inter scale product and noise variance. 
In this study, a new method is proposed to determine the mask by identifying the degree of significance of each coefficient. The degree of significance is determined using a dependency measure based on the product of wavelet coefficients at adjacent scales. It is then compared against the signal variance.

The rule for the determination of mask is given by,

$$
\hat{I}_{i, j}=\left\{\begin{array}{ll}
0 & \left|\mathrm{~W}_{\mathrm{i}, \mathrm{j}}\right|\left|W_{i+1, j+1}\right| \prec \sigma_{x}^{2} \\
1 & \left|\mathrm{~W}_{\mathrm{i}, \mathrm{j}}\right|\left|W_{i+1, j+1}\right| \geq \sigma_{x}^{2}
\end{array},\right.
$$

where $W_{i j}$ is the wavelet coefficient at scale $j$ and $W_{i+1, j+1}$ is the wavelet coefficient at scale $j+1 . \sigma_{x}^{2}$ is the signal variance estimated using a local neighbourhood as given in Eq. (4) for the subband under consideration.

$$
\sigma_{x}^{2}=\frac{1}{M} \sum_{m=1}^{M} x_{m}^{2}
$$

The detection of mask is done starting from the coarsest level of decomposition to the finest level. The classification is performed for all the detail subbands. The resultant mask gives a measure for the locations of signal of interest for each detail image. Each detail subband is then multiplied with its corresponding mask and the resultant subband matrix is used in the estimation of signal variance to determine the threshold.

2.2b Estimation of signal variance exploiting intra scale dependency: The estimation of signal variance is a critical issue in any denoising scheme. It is estimated generally by using a local window around the neighbourhood of each pixel using Maximum Likelihood (ML) or Maximum A Posteriori (MAP) estimate. The absolute value or the squared values of neighbouring coefficients are correlated. The averages are useful in collecting information about other coefficients in the near vicinity (Kim \& Ra 2005; Kumar 2005). This procedure does not provide much information about the spatial adaptivity of each pixel.

The information about the homogenous neighbours in a local window is useful in preserving the important image details (Chen et al 2009). Hence a measure of local spatial adaptivity for each pixel position is included in the estimation of signal variance in this study.

This study proposes a truncation threshold to determine the homogenous neighbours by exploiting the intra scale dependency of the coefficients in each subband. The truncation threshold $(\phi)$ is calculated from the sample mean absolute deviation of the data from their mean as,

$$
\phi=\frac{1}{n} \sum_{i=1}^{n}\left(\left|x_{i}-\mu\right|\right)
$$

where $\mu$ is the mean of the sub-band under consideration and $n$ is the number of pixels in the subband. The wavelet coefficients at different resolution scales are compared against this threshold and a weight kernel is estimated as given in Eq. (6)

$$
W_{T}(i, j)=\left\{\begin{array}{ll}
0 & x(i, j) \prec \phi \\
1 & x(i, j) \geq \phi
\end{array} .\right.
$$

The estimated weight kernel serves as a local spatial activity indicator for the pixels in the subband. A finite dataset $x_{i}(i=1 \ldots . . N)$ can be truncated to two subsets, one set containing large magnitude data and the second set containing smaller magnitude data, with the help of the mean 
of the data set. Hence the absolute deviation of each data in the set from their mean and the average of all such deviations serve as a threshold in determining the significant data. Thus, the mean absolute deviation of the pixels from their calculated subband mean gives an indication of the presence of an edge pixel (large magnitude pixel). Thus it helps in retaining the important image details.

Chang et al (2000a, b) proposed a spatially adaptive threshold based on context modelling, where the statistical modelling for a given coefficient was carried as a function of its neighbours. Context modelling classifies the coefficients into several classes based on different values of $\sigma_{x}$. Classification is performed by taking the weighted average of the coefficients located in a casual neighbourhood and it falls within a specified range.

In the proposed method, statistical estimates of the coefficients are calculated by a more flexible method. The variance of the subband coefficients are calculated using the weight kernel determined from Eq. (6). The estimation of the proposed signal variance is as follows:

$$
\begin{gathered}
\sigma_{x}=\sqrt{\max \left(\sigma_{y}^{2}-\sigma_{n}^{2}\right), 0}, \\
\sigma_{y}^{2}=\frac{1}{M N} \sum_{i=1}^{M} \sum_{j=1}^{N} Y_{k}(i, j) \bullet W_{T}(i, j) .
\end{gathered}
$$

$\mathrm{MN}$ is the size of the image and $Y_{K}$ is the sub-band and $W_{T}$ is the weight kernel. The signal variance thus estimated gives a good indication of the local variability of the coefficients. The procedure is repeated for all the subband coefficients to calculate their signal variance and hence the threshold for all the subbands.

2.2c Proposed thresholding function: The traditional hard and soft thresholding have the following shortcomings. In the hard thresholding approach, the denoised wavelet coefficients show discontinuity on threshold $\lambda$ and $-\lambda$, resulting in Gibbs shock. In the soft-thresholding method, there is no discontinuity. But there exists a constant bias between the denoised coefficients and the original coefficients, when the processed coefficient is greater than the threshold. The result is that the original features could not be maintained.

In order to improve the denoising performance, the fixed bias may be reduced, but cannot be reduced to zero (Hard Thresholding Function). Qin et al (2010) proposed an improved adaptive threshold function to reduce the fixed bias of soft thresholding by estimating the coefficients between $\left|W_{j, k}\right|$ and $W_{j, k}$. But still the magnitude of the reconstructed coefficient does not approach the original coefficient's magnitude in their approach.

The proposed approach aims to enhance the improved adaptive thresholding function of Qin et al (2010), in two folds. One possible improvement is in the direction of reducing further the fixed bias of the reconstructed coefficients and the second by reducing the number of zero coefficients.

The proposed new and improved adaptive threshold function is defined as,

$$
\begin{aligned}
& \hat{W}_{j, k}=\operatorname{sgn}\left(W_{j, k}\right)\left(\left|W_{j, k}\right|-\left(\lambda_{j}-\exp \left(\frac{-p}{\left|W_{\max }\right|^{2}-\lambda_{j}^{2}}\right) \lambda_{j}\right)\right) \text { for } \quad\left|W_{j, k}\right| \geq \lambda_{j} \\
& =W_{j, k-\mu_{j, k}} \quad \text { for } \quad \frac{\lambda_{j}}{3} \leq\left|W_{j, k}\right| \prec \lambda_{j} \\
& =0 \quad \text { otherwise }
\end{aligned}
$$


where $p$ is a positive constant. In the above equation $\widehat{W}_{j, k}$ is the denoised wavelet coefficient, $W_{j, k}$ is the noisy wavelet coefficient, $\lambda_{j}$ is the subband adaptive threshold, $W_{\max }$ is the maximum wavelet coefficient of the subband under consideration and $\mu_{j, k}$ is the mean estimator determined for individual coefficients in each subband by considering a local neighbourhood.

The exponential weighting function, in Eq. (9) helps in reducing the fixed bias. For $\left|W_{j, k}\right| \geq \lambda_{j}$, the adaptive thresholding function approximates soft thresholding function When $p \rightarrow \infty$ and approximates hard thresholding when $p \rightarrow 0$. The proper selection of $\mathrm{p}$ improves

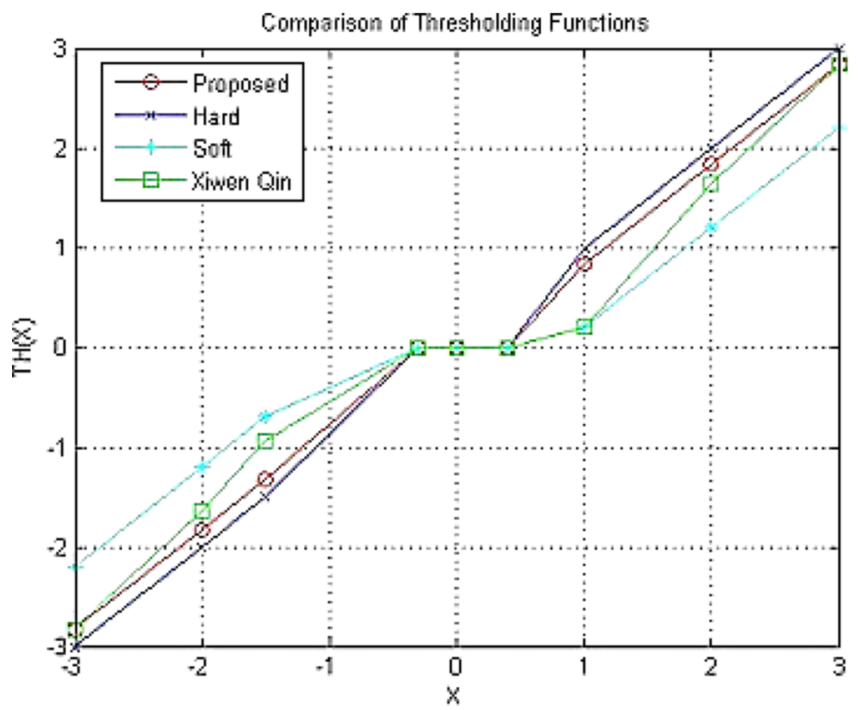

Figure 3. Comparison of thresholding function for $\left|W_{j, k}\right| \geq \lambda_{j}$.

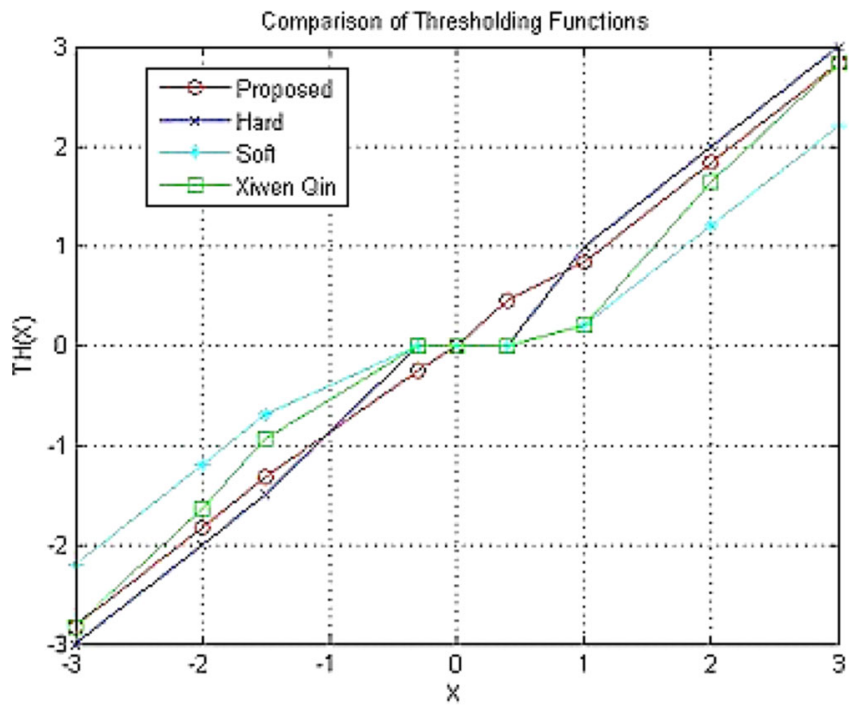

Figure 4. Comparison of Eq. (6) with other thresholding functions. 
the denoising performance. The parameter $W_{\max }$ in the denominator of the exponential weighting function helps in further reducing the fixed bias of the reconstructed coefficient.

The property of the proposed thresholding function can be verified from figures 3 and 4 .

From figure 3, it is seen that the proposed thresholding function reduces the fixed bias of the soft thresholding function better than (Qin et al 2010). Figure 4 shows that the proposed thresholding function in Eq. (6) reduced the zero coefficients. The reduction in fixed bias and the zero coefficients aid in keeping the important features of the original image, thus providing an improved noise reduction performance.

$2.2 \mathrm{~d}$ Determination of subband adaptive threshold: If the threshold $\lambda$ is a constant parameter then it will result in too many kill of the wavelet coefficients. Therefore, the threshold $\lambda_{j}$ is selected to be subband adaptive in this study.

The subband adaptive threshold is given by

$$
\lambda_{j}=\beta T_{B},
$$

where $\beta$ is a subband adaptation parameter.

$$
\beta=\sqrt{\frac{\log M}{2^{j}}}
$$

$\mathrm{M}$ is the total no. of coefficients and $j$ is the current level of decomposition $T_{B}$ is the Bayes threshold given by

$$
\begin{aligned}
T_{B} & =\frac{\sigma_{n}^{2}}{\sigma_{x}} & & \text { if } \quad \sigma_{n}^{2} \prec \sigma_{x}^{2}, \\
& =\max \left|Y_{i, j}\right| & & \text { Otherwise. }
\end{aligned}
$$

$\sigma_{x}^{2}$ is the signal variance and $\sigma_{n}^{2}$ is the noise variance estimated using the robust median estimator of the highest subband of the wavelet transform.

$$
\sigma_{n}=\text { median }\left(\left|Y_{i, j}\right| / 0.6745\right) \text {. }
$$

Thus the subband adaptive threshold $\lambda_{j}$ removes noisy coefficients simultaneously preserving the important image details.

The proposed wavelet denoising algorithm is summarized as follows:

- Compute the stationary wavelet transform for the input image to the required $\mathrm{J}$ resolution levels

- For each scale of decomposition $2^{j}, \mathrm{j}=1 \ldots \mathrm{J}-1$

- For each orientation (HL,LH,HH), perform the following :

- Determine the mask, $\hat{I}_{i, j}$ using inter-scale dependency starting from the coarsest subband to finest subband using Eq. (3)

- Multiply each detail subband with its corresponding mask

- Compute noise variance using Eq. (13)

- Compute the signal variance using Eq. (7) by

* Computing the truncation threshold using Eq. (5)

* Calculating the weight kernel using Eq. (6)

* Computing $\sigma_{y}^{2}$ as in Eq. (8) 
- Calculate the threshold $T_{B}$ using Eq. (12)

- Apply thresholding using the shift invariant improved adaptive thresholding function as in Eq. (9)

- Take inverse stationary wavelet transform

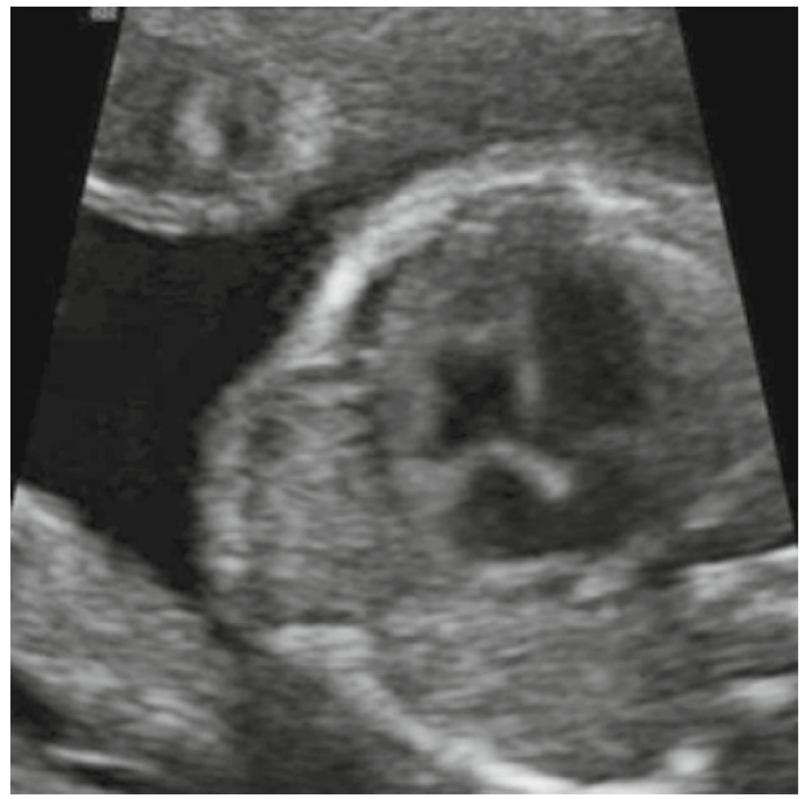

Figure 5. Ultrasound image 1.

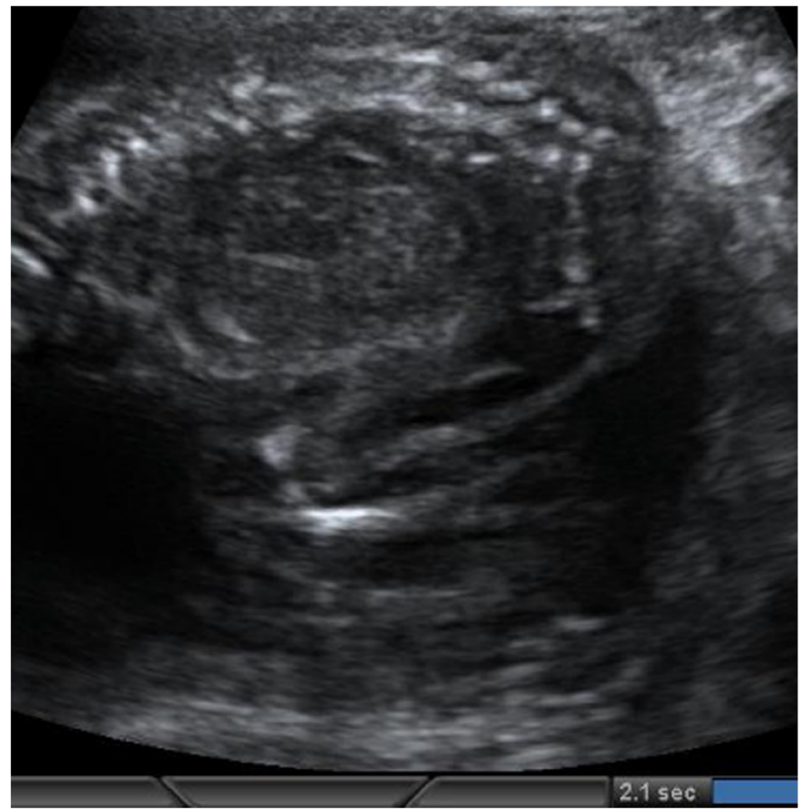

Figure 6. Ultrasound image 2. 


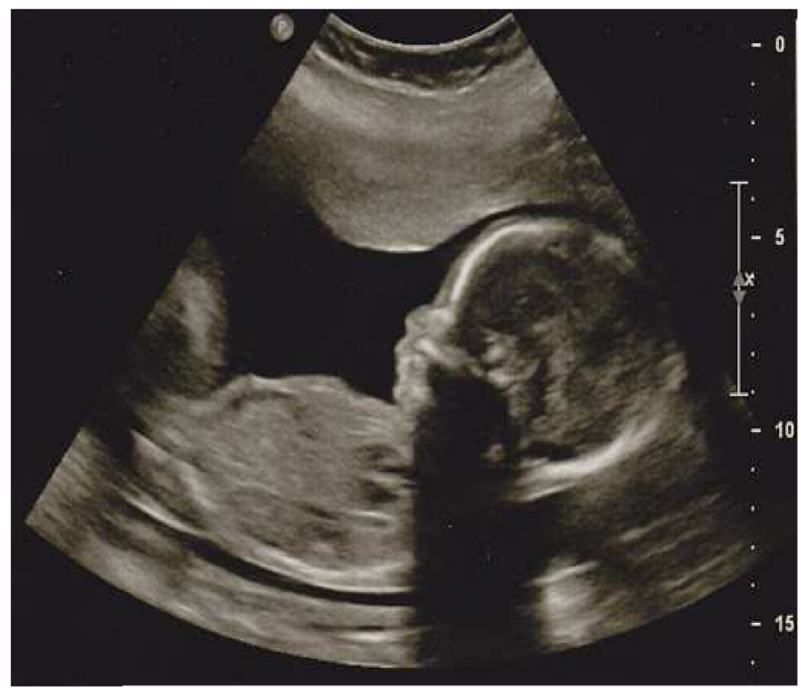

Figure 7. Ultrasound image 3.

\section{Results and discussion}

Experiments were conducted on the clinical ultrasound images of size $512 \times 512$. The ultrasound images are obtained from Edapal Hospital, Kerala. The images used in this experiment are shown in figures 5, 6 and 7 .

The proposed approach (SIIATF) is compared with the existing spatial filters like Lee, Frost, Kuan, wavelet denoising using soft thresholding (Bayesshrink), Shift invariant wavelet denoising using Bayesian shrink (SIshrink) and with the results of Qin et al (2010). Results were generated for various noise variances, for different wavelet bases and for varying window sizes $(3 \times 3$, $5 \times 5$ and $7 \times 7$ ). Quantitative performance was measured with parameters like Peak Signal to Noise ratio (PSNR), Mean Square Error (MSE), Structural Similarity Index Measure (SSIM), Equivalent Number of Looks (ENL) and Edge preservation Index (EPI) with formulas given below:

$$
\begin{gathered}
P S N R=10 \log _{10}\left(\frac{255^{2}}{M S E}\right) \quad d B, \\
M S E=\frac{1}{M N} \sum_{i=1}^{M} \sum_{j=1}^{N}(X(i, j)-P(i, j))^{2}, \\
E N L=\frac{N M V^{2}}{N S D^{2}} \\
N S D=\frac{1}{M N} \operatorname{sort} \sum_{i=1}^{M} \sum_{j=1}^{N}\left(X_{i, j-N M V}\right)^{2}, \\
N M V=\frac{1}{M N} \sum_{i=1}^{M} \sum_{j=1}^{N} X_{i, j},
\end{gathered}
$$


央

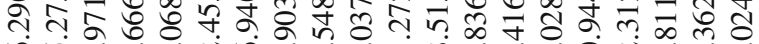

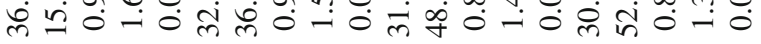

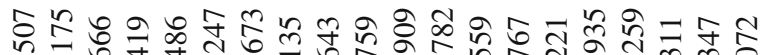
กิ 们

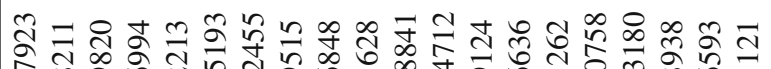

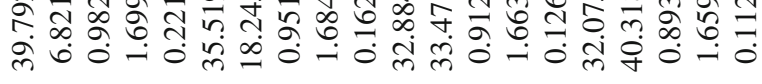
๙ 蚛苂

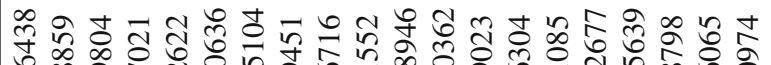

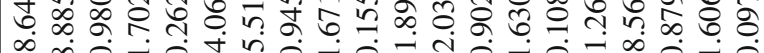

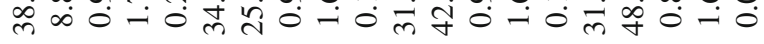

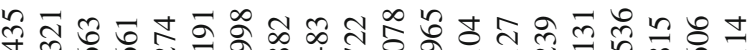

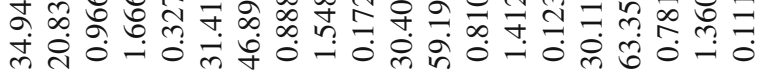

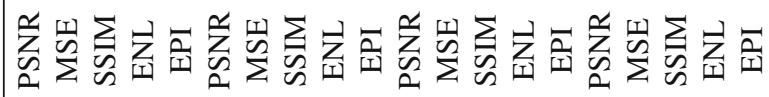




$$
\begin{gathered}
\operatorname{SSIM}(x, y)=\frac{\left(2 \mu_{x} \mu_{y}+C_{1}\right)\left(2 \sigma_{x, y}+C_{2}\right)}{\left(\mu_{x}^{2}+\mu_{y}^{2}+C_{1}\right)\left(\sigma_{x}^{2}+\sigma_{y}^{2}+C_{2}\right)}, \\
E P I=\frac{\Sigma\left(\nabla I\left(x_{i}-\overline{\nabla I}\right)\right)\left(\overline{\nabla \hat{I}} x_{i}-\overline{\nabla \hat{I}}\right)}{\sqrt{\Sigma}\left(\nabla I\left(x_{i}-\overline{\nabla I}\right)\right)^{2} \Sigma\left(\overline{\nabla \hat{I}} x_{i}-\overline{\nabla \hat{I}}\right)^{2}},
\end{gathered}
$$

Table 2. Comparison of various wavelet basis for ultrasound image 1 .

\begin{tabular}{lcccr}
\hline Parameters & $\mathrm{dB} 4$ & Coif3 & Sym2 & Coif2 \\
\hline PSNR $(d B 4)$ & 42.2175 & 41.8756 & 42.7856 & 29.4589 \\
MSE & 3.9024 & 4.9878 & 4.5689 & 41.5987 \\
SSIM & 0.9854 & 0.9852 & 0.9850 & 0.9849 \\
EPI & 0.5979 & 0.5678 & 0.5489 & 0.2678 \\
ENL & 1.7131 & 1.7165 & 1.7152 & 1.7789 \\
\hline
\end{tabular}

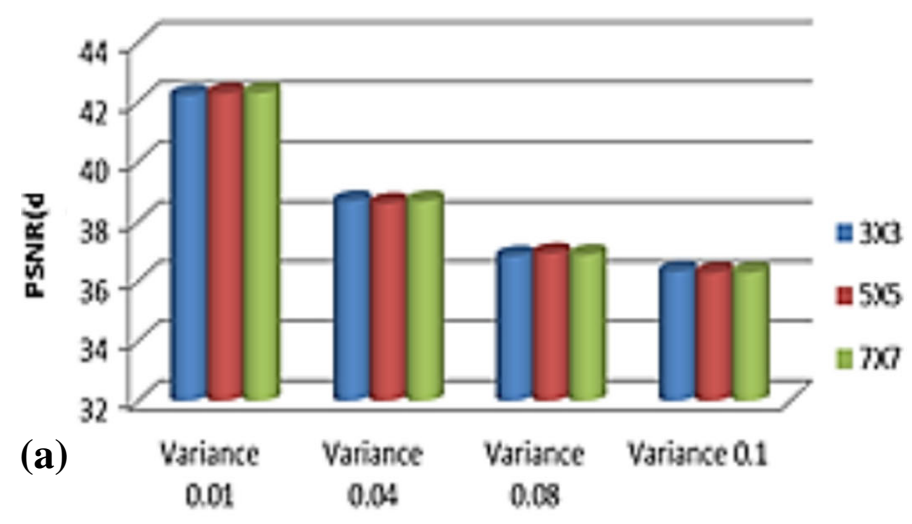

\section{Noise variance}

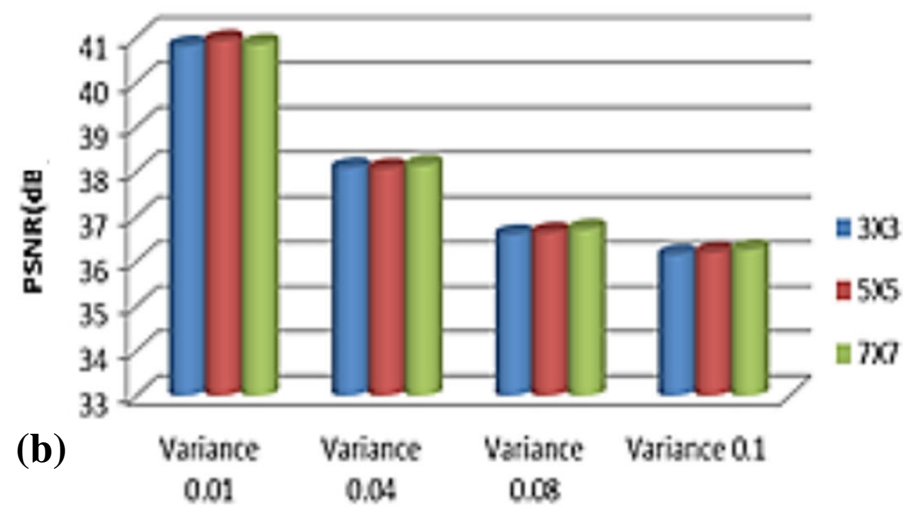

\section{Noise variance}

Figure 8. Comparison of PSNR for various window sizes for ultrasound images. 
where $X(i, j)$ is original image, $P(i, j)$ is denoised image, $\mu_{x}$ is mean of original image, $\mu_{y}$ is mean of denoised image, $\sigma_{x}^{2}$ is variance of original image, $\sigma_{y}^{2}$ is variance of denoised image, $\nabla I$ is the gradient of the original image and $\nabla \hat{I}$ is the gradient of the denoised image. The results of comparison of the performance metrics of the proposed approach against several existing filters as mentioned above and for various noise variances are furnished in table 1 . The results given in table 1 indicate the performance metrics of the filter tested with ultrasound image 1.

The results shown in table 1 were generated using db4 wavelet with a window size of $3 \times 3$. The analysis of different wavelet basis and of varying window sizes $(3 \times 3,5 \times 5$ and $7 \times 7)$ is furnished in table 2 and in figure 8.

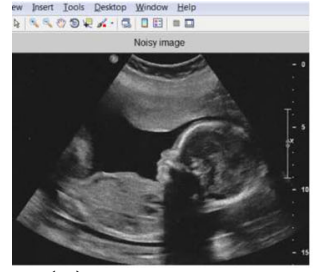

(a) Noisy Image $\left(\sigma^{2}=0.01\right)$

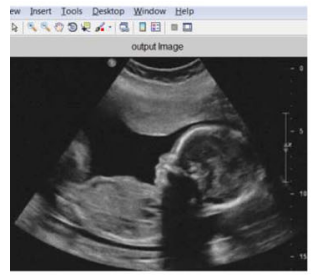

(e) Bayes shrink output

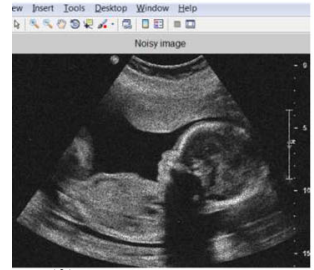

(i) Noisy Image $\left(\sigma^{2}=0.1\right)$

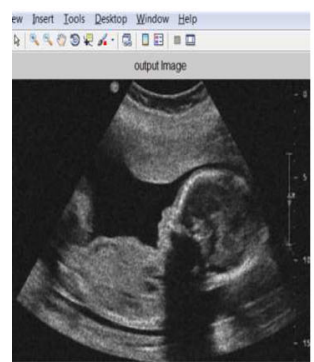

(m) Bayes shrink output

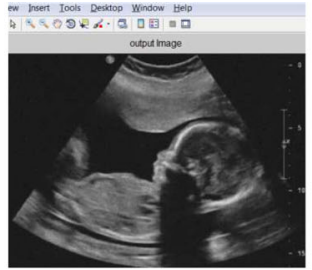

(b) Frost Filter output

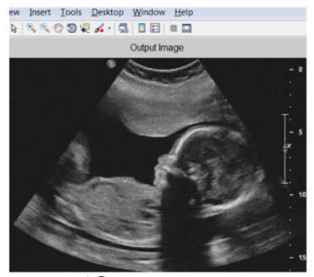

(f) SIshrink

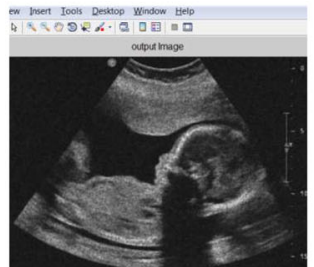

(j) Frost Filter output

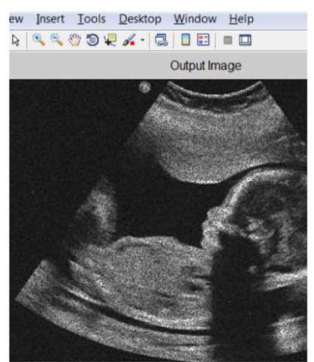

(n) SIshrink

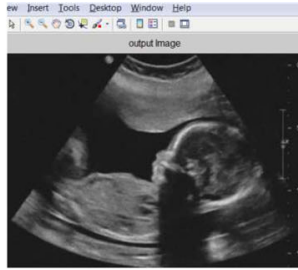

(c) Lee Filter output

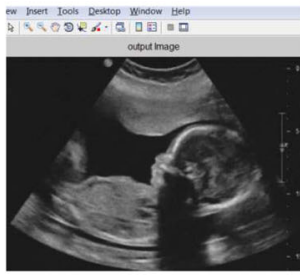

(g) Xiwen Qin

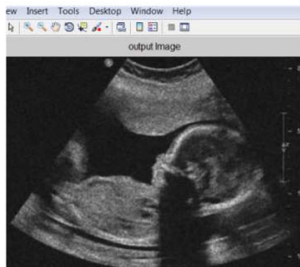

(k) Lee Filter output

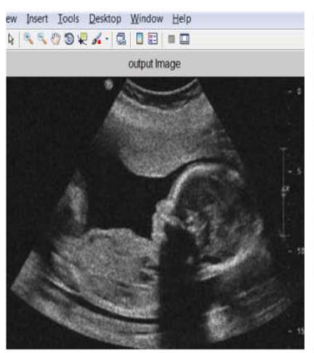

(o) Xiwen Qin

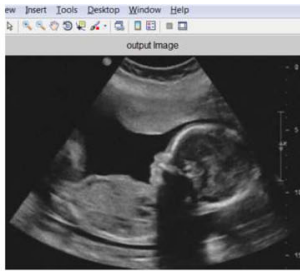

(d) Kuan Filter output

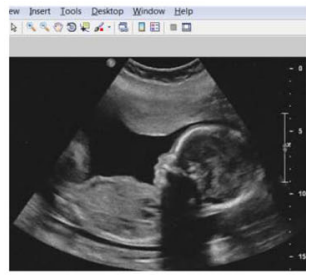

(h) SIIATF (Proposed)

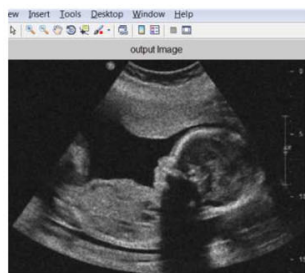

(1) Kuan Filter output

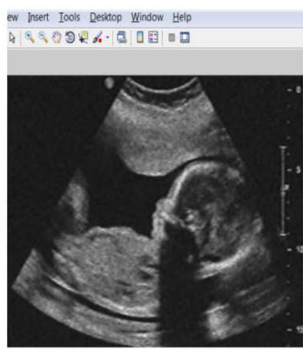

(p) SIIATF (Proposed)

Figure 9. Output images for ultrasound image 3. 
Figure 8 shows the comparison of PSNR for ultrasound image 1 and ultrasound image 2 for different noise variances with varying window sizes. The improvement in the visual quality of the proposed (SIIATF) approach is compared with the existing filters in figure 9 for Ultrasound image 3 for $\sigma^{2}=0.01$ (a to h) and $\sigma^{2}=0.1$ (i to $\mathrm{p}$ ).

Figure 10 shows the visual quality improvement of the proposed filter (SIIATF) of ultrasound image 1 for four different noise variances.

The first row of figure 10 shows the noisy images and the second row shows the output of the SIIATF. The performance measures for ultrasound image 2 are analysed graphically in figure 11 .

From the tables and graphs, it is seen that the proposed filter performs better for different noise variances. With the new shift invariant improved adaptive thresholding function, the filter shows a significant improvement in PSNR and reduction in MSE value. PSNR is increased in the range of 1.8 to $2.4 \mathrm{~dB}$ from low to high noise variance. For the input image 2, the filter performed well at higher noise variances and PSNR is increased up to $1.5 \mathrm{~dB}$. The parameter MSE had been phenomenally reduced for both the images. SSIM and ENL measures have also increased. Another important parameter that shows a significant improvement is the Edge Preservation Index (EPI). EPI is a measure of how well the edges and fine details are preserved in the denoised image.

The preliminary classification of signal of interest and noise coefficients exploiting inter-scale dependency of the wavelet coefficients significantly improves the performance of the proposed filter. It narrows down the coefficients to be processed by considering only the significant image objects. The estimation of weighted signal variance serves as a local spatial adaptivity indicator for the coefficients within the subband. The truncation threshold stated for the determination of weight kernel thus helps in retaining the important image features. This aids in retaining the

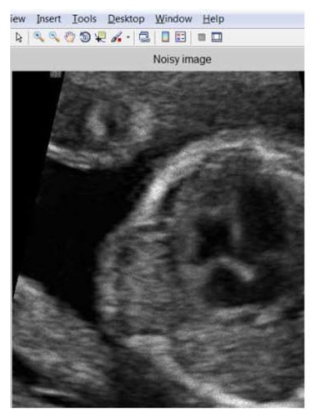

$\sigma^{2}=0.01$

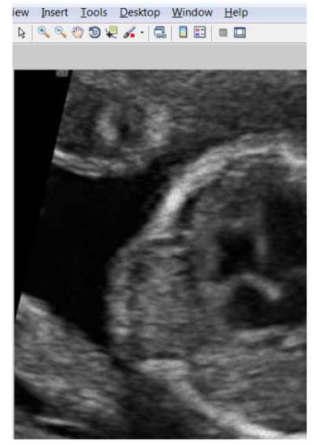

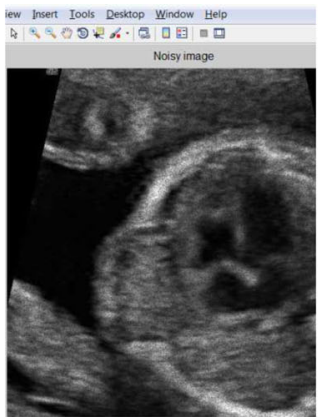

$\sigma^{2}=0.04$

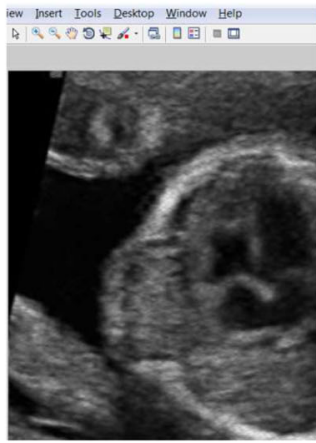

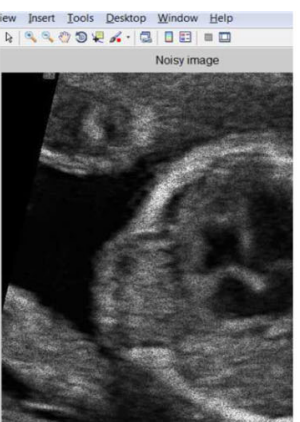

$\sigma^{2}=0.08$

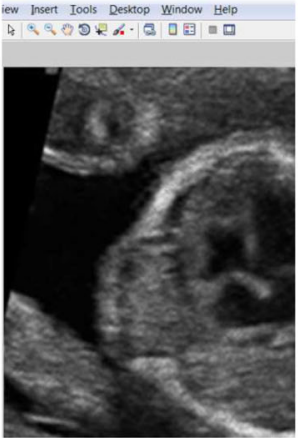

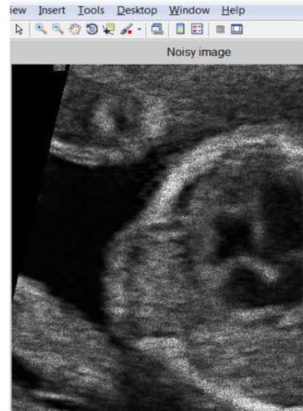

$\sigma^{2}=0.1$

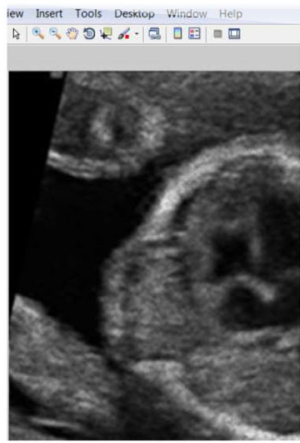

Output of Proposed SIIATF

Figure 10. Output images for ultrasound image 1 using proposed method. 


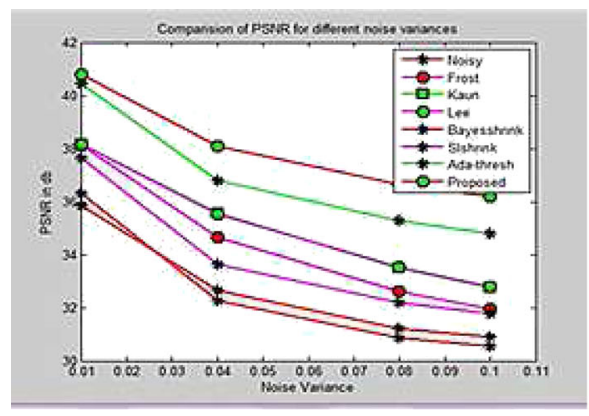

(a). Comparison of PSNR for different noise variances.

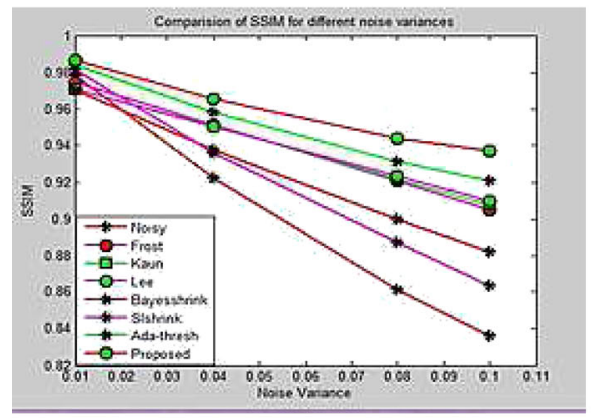

(c). Comparison of SSIM for different noise variances.

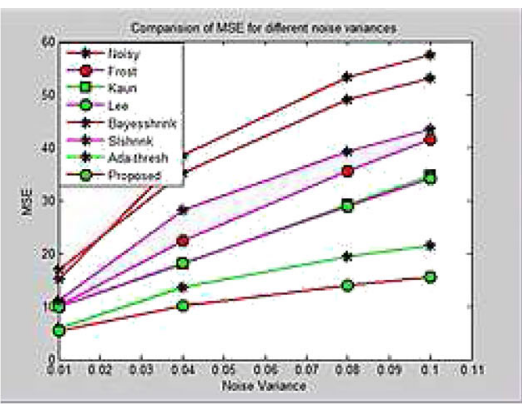

(b). Comparison of MSE for different noise variances.

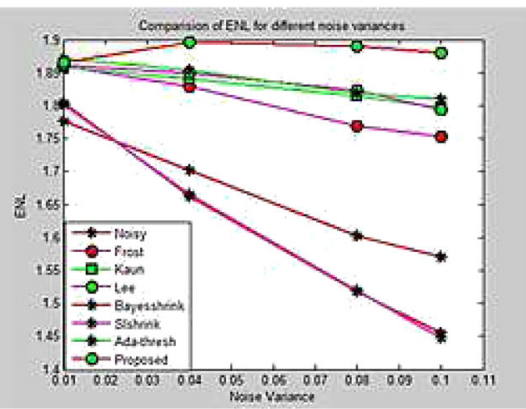

(d). Comparison of ENL for different noise variances.

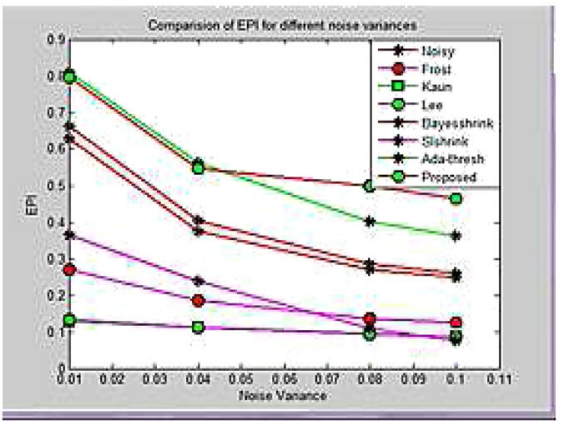

(e). Comparison of EPI for different noise variances.

Figure 11. Comparison of performance measures for ultrasound image 2.

original image features in the reconstructed image and hence an improved edge preservation is achieved. On an average, for the two input images, EPI was increased up to $3.3 \%$ for low noise variance and up to $35 \%$ for high noise variance. The results generated for various noise variances with different wavelets are shown in table 2. The PSNR value is high for Sym 2 compared to other wavelet basis. But SSIM and EPI parameters are high for $\mathrm{dB} 4$ wavelets with a reduction in MSE. Thus dB4 preserves more edges and fine details with a better PSNR for the given images. In this paper, the results are generated with $\mathrm{dB} 4$ wavelets. The results for varying window sizes indicate that at lower noise variances a small window is sufficient for taking the local neighbourhood and for higher noise variances an increase in window size yields a better result. The results 
and comparisons indicate that the proposed approach performs better than the existing filtering approaches in noise removal as well as in preserving edges and fine details.

\section{Conclusion}

The proposed approach uses a shift invariant improved adaptive wavelet threshold function (SIIATF) for the removal of speckle noise in ultrasound images. The preliminary classification performed is based on the persistence of the important image features across the scales and it helps in retaining the edges and fine details. The sample mean absolute deviation of the data from their mean gave a good indication of the local variability of the coefficients. Hence, the weighted variance estimate provided a better adaptation to the threshold and resulted in a good edge preservation index. The exponential weighting function helps in reducing the fixed bias of soft thresholding approach. The performance of the filter was better when simulated with dB4 wavelet. From the performance analysis discussed in the previous section, it is seen that the filter achieved a comparatively better performance in terms of PSNR and EPI at high noise variances. Thus, the proposed approach produced better denoising results both in terms of visual quality and quantity.

\section{References}

Achim A, Bezerianos A and Tsakalides P 2001 Novel bayesian multiscale method for speckle removal in medical ultrasound images. IEEE Trans. Med. Imaging 20(8): 772-783

Arsenault H H and April G 1976 Properties of speckle integrated with a finite aperture and logarithmically transformed. J. Opt. Soc. Am.1160-1163

Bhuiyan M I H, Ahmad M O and Swamy M N S 2009 Spatially adaptive thresholding in wavelet domain for despeckling of ultrasound images. IET Image Process. 3(3): 147-162

Chen $\mathrm{P}$ and Suter D 2004 Shift-invariant wavelet denoising using interscale dependency. IEEE Int. Conf. Image Process. 2: 1005-1008

Chen Y, Ji Z-C and Hua C-J 2009 Efficient statistical modeling of wavelet coefficients for image denoising. Int. J. Wavelets Multiresolution Inf. Process. 7(5): 629-641

Coifman R R and Donoho D L 1995 Translation-invariant de-noising, in Wavelets and Statistics Lecture Notes in Statistics 103: 125-150

Donoho D L 1995 De-noising by soft thresholding. IEEE Trans. Inf. Theory 41(3): 613-627

Gao H-Y 1998 Wavelet shrinkage denoising using the non-negative garrotte. J. Comput. Graph. Stat. 7(4): 469-488

Goodman J W 1976 Some fundamental properties of speckle. J. Opt. Soc. Am. 66: 1145-1150

Grace Chang S, Yu B and Vetterli M 2000a Spatially adaptive wavelet thresholding with context modeling for image denoising. IEEE Trans. Image Process. 9(9): 1522-1531

Grace Chang S, Yu B and Vetterli M 2000b Adaptive wavelet thresholding for image denoising and compression. IEEE Trans. Image Process. 9(9): 1532-1546

Gyaourova A, Kamath C and Fodor I K 2002 Undecimated wavelet transforms for image de-noising. DOI:10.1.1.8.1435

Ismail B and Khan A 2012 Image de-noising with a new threshold value using wavelets. J. Data Sci. 10: $259-270$

Kalaivani Narayanan S and Wahidabanu R S D 2009 A view of despeckling in ultrasound imaging. Signal Processing. Image Process. Pattern Recog. 3: 85-98

Kaur J, Kaur J and Kaur M 2011 Survey of despeckling techniques for medical ultrasound images. Int. J. Comp. Tech. Appl. 2(4): 1003-1007 
Kim Y S and Ra J B 2005 Improvement of ultrasound image based on wavelet transform: speckle reduction and edge enhancement. Medical Imaging: Image Processing, Proc. of SPIE Vol. 5747

Kumar S 2005 Performance Improvement in the Bivariate models by using modified marginal Variance of noisy observations for Image denoising applications. World Academy of Science. Eng. Technol. 11: 103-107

Liu J and Moulin P 2001 Information-theoretic analysis of interscale and intrascale dependencies between image wavelet coefficients. IEEE Trans. Image Process. 10(11): 1647-1658

Matsuyama E, Tsai D-Y, Lee Y, Tsurumaki M, Takahashi N, Watanabe H and Chen H-M 2012 A modified undecimated discretewavelet transform based approach to mammographic image denoising. J. Digit. Imaging 26(4): 748-758

Pižurica A, Philips W, Lemahieu I and Acheroy M 2001 Despeckling SAR images using wavelets and a new class of adaptive shrinkage functions. Proceedings of IEEE Int. Conference on Image Processing, ICIP 2001, Thessaloniki, Greece

Pižurica A, Philips W, Lemahieu I and Acheroy M 2002 A joint inter- and intrascale statistical model for bayesian wavelet based image denoising. IEEE Trans. Image Process. 11(5): 545-557

Pižurica A, Philips W, Lemahieu I and Acheroy M 2003 A versatile wavelet domain noise filtration technique for medical imaging. IEEE Trans. Med. Imaging 22(3): 323-331

Puvanathasan P and Bizheva K 2007 Speckle noise reduction algorithm for optical coherence tomography based on interval type II fuzzy set, Vol. 15, No. 24/Optics Express 15(24): 15747-15758

Qin X, Yue Y, Dong X, Wang X and Tao Z S 2010 An improved method of image denoising based on wavelet transform. International Conference on Computer, Mechatronics, Control and Electronic Engineering (CMCE)

Sadler B M and Swami A 1999 Analysis of multiscale products for step detection and estimation. IEEE Trans. Inf. Theory 45(3): 1043-1051

Sudha S, Suresh G R and Sukanesh R 2009 Speckle noise reduction in ultrasound images by wavelet thresholding based on weighted variance. Int. J. Comput. Theory Eng. 1(1): 1793-8201

Suetens P 2002 Fundamentals of Medical Imaging, 1st Edition, Cambridge University, UK 145-182

Viswa A and Sharma S 2012 Modified method for denoising the ultrasound images by wavelet thresholding. I. J. Intell. Syst. Appl. 6: 25-30

Xie H, Pierce L and Ulaby F T 2002 Statistical properties of logarithmically transformed speckle. IEEE Trans. Geosci. Remote Sens. 40: 721-727

Xu J, Zhang K, Xu M and Zhou Z 2009 An adaptive threshold method for image denoising based on wavelet domain, MIPPR 2009: Automatic Target Recognition and Image Analysis, Proc. of SPIE Vol. 7495

Yang J, Fengb R and Deng W 2011 A New Algorithm of Image Denoising Based on Stationary Wavelet Multi-scale Adaptive Threshold, Proceedings, International conference on Electronic \& Mechanical and Information Technology, 12-14 August, 4550-4553

Yoo Y, Ortega A and Yu B 1999 Image subband coding using context based classification and adaptive quantization. IEEE Trans. Image Process. 8: 1702-1715

Zhang D, Bao P and Wu X 2005 Multiscale LMMSE-based image denoising with optimal wavelet selection. IEEE Trans. Circ. Syst. Video Technol. 15(4): 469-481 